Archived version from NCDOCKS Institutional Repository http://libres.uncg.edu/ir/asu/

\title{
Appalachỉan
}

B O O N E, N O R T H C A R O L I N A

\section{Stage Transition And Laryngeal Closure In Poststroke Patients With Dysphagia}

\author{
By: Elizabeth Rachel Oommen • Youngsun Kim • Gary McCullough
}

\begin{abstract}
Timely hyolaryngeal excursion and laryngeal closure are essential for safe transfer of the bolus during the pharyngeal swallow. The temporal measures stage transition duration (STD) and laryngeal closure duration (LCD) represent these physiological events. The purpose of this investigation was to determine whether small changes in bolus consistency and volume affect these temporal measures in poststroke patients who aspirate, poststroke patients who do not aspirate, and nonneurologically impaired control subjects. STD and LCD were obtained by frame-by-frame analysis of the videofluoroscopic examinations of 5 and $10 \mathrm{ml}$ thin and nectar thick liquids. Using a three-way analysis of variance, STD was significantly different between the control group and the two poststroke groups; however there was no difference between the two stroke groups. There was no difference among the three groups on LCD. Bolus consistency and bolus volume had no effect. Clinical implications of these findings are discussed.
\end{abstract}

Elizabeth Rachel Oommen • Youngsun Kim • Gary McCullough (2011) "Stage Transition And Laryngeal Closure In Poststroke Patients With Dysphagia" Dysphagia 26:318-323 Version of Record Available At www.springer.com [DOI 10.1007/s00455-010-9314-0] 


\section{$\underline{\text { Stage Transition and Laryngeal Closure in Poststroke Patients }}$ withDysphagia}

Swallowing involves the transfer of food or liquid through the oral, pharyngeal, and esophageal stages into the stomach through the gastroesophageal junction [1]. In poststroke patients, an increased incidence of oropharyngeal dysphagia has been associated with risk of aspiration pneumonia and decreased quality of life. Radiographic swallowing examinations have been reported to identify dysphagia in $64-78 \%$ of poststroke patients [2]. Objective data obtained from radiographic evaluations are useful in understanding the physiological impairments in poststroke patients with dysphagia [3]. The temporal measures obtained from radiographic swallowing examinations represent specific physiological events associated with airway protection and the transfer of the bolus into the esophagus and help refine diagnostic tools, understand disordered physiological events, and develop effective treatment plans for dysphagia [4].

Stage transition duration (STD) represents the transition between the oral and pharyngeal stages of swallowing and the timely initiation of the pharyngeal swallow. STD is calculated as the time difference between two events in pharyngeal swallowing: bolus head passing the ramus of mandible and the initiation of maximum hyoid excursion [5]. In younger healthy individuals, STD values were found to be zero or negative [5], which indicates that the initiation of hyoid excursion occurred prior to the bolus passing the ramus of mandible. However, STD has been found to increase with age $[3,5]$. Penetration, which refers to the entry of the bolus into the larynx above the level of the vocal folds [1], and aspiration, which refers to the entry of the bolus into the airway and passing below the vocal folds [1], may be related to prolonged STD values secondary to delayed response of pharyngeal musculature [5]. STD values have correctly identified the presence of aspiration $75 \%$ of the time in poststroke patients [6]. 
Aspiration can also result from inadequate and incomplete laryngeal closure [1]. Laryngeal closure duration (LCD) is the duration of contact between the arytenoids and the epiglottis [7]. LCD reflects the integrity of an airway protection mechanism that prevents the entry of the bolus into the airway during the swallow. Poststroke patients have been shown to exhibit significantly shorter LCDs than healthy normal subjects [8].

Research suggests that changes in bolus characteristics can influence the timing of physiological events in swallowing [7, 9-12]. Healthy individuals exhibited increased STD values when bolus consistency was changed from liquid to semisolid [5]. When the bolus consistency was changed from liquid to pudding, healthy individuals exhibited reduced pharyngeal response times and durations of laryngeal elevation, while poststroke patients exhibited shorter pharyngeal delay times [9]. The timing of physiological events in pharyngeal swallowing are influenced by changes in bolus volume as well [4]. Increased bolus volumes result in earlier elevation of the soft palate and aryepiglottic folds and increased duration of the upper esophageal sphincter (UES) opening. Bolus volume also affected LCD [7] and pharyngeal response time in healthy normal individuals [9]. Effects of different bolus consistencies and volumes on the timing of physiological events may have therapeutic implications in poststroke patients exhibiting reduced range of hyolaryngeal excursion [9].

There have been a limited number of investigations, to our knowledge, that focused on the effects of thickening liquids on the timing of physiological events in poststroke patients with dysphagia. The purpose of this investigation was to examine whether small changes in bolus consistency (thin vs. nectar thick) and bolus volume (5 vs. $10 \mathrm{ml}$ ) would affect the temporal measures of STD and LCD in elderly nonneurologically impaired healthy subjects (control group), poststroke patients who did not exhibit aspiration (nonaspirators), and poststroke patients who did exhibit aspiration (aspirators). Knowledge about these effects could facilitate early identification of dysphagia in poststroke patients and thereby prevent further health complications.

\section{Method}

\section{Subjects}

Sixty stroke patients had videofluoroscopic swallowing examinations (VFSEs) for a prior investigation [13], of which 22 patients exhibited aspiration. Forty-four patients suffered a single stroke while 16 patients had two or more strokes. Twenty-nine of the 60 patients had cortical lesions, of which 11 were in the right hemisphere, 17 were in the left hemisphere, and 1 was bilateral. Fourteen patients had subcortical strokes and six patients had brainstem strokes. Three patients had cerebellar lesions, three had mixed localization, and three could not be localized.

For this investigation, 52 patients were chosen based on the clarity of the pharyngeal structures in the VFSEs. Only the highest quality VFSEs were included to improve the accuracy of temporal measurements. Among the 52 poststroke patients, 19 exhibited aspiration (aspirators) and 33 did not (nonaspirators). The mean age of the stroke patients was 67.5 years. Patients were determined to show aspiration if the bolus passed the level of the true vocal folds in at least one of the swallows. Aspiration may not have occurred on all the same bolus volumes/consistencies.

Twenty healthy nonneurologically impaired subjects who had VFSEs from a previous investigation [14] served as the control group. All subjects were screened for neurological and structural abnormalities that could affect their deglutition and completed comprehensive questionnaires as well as oral motor and neurological exams. The mean age of the control group was 68.75 years.

\section{Videofluoroscopic Swallowing Examination Procedure}

The VFSEs were collected using the same methodology for the poststroke patients and for the control group [13, 14]. A stretcher chair was used to seat the patient upright during the course of the examination. VFSEs were collected by using a mobile $\mathrm{C}$-arm X-ray machine and were recorded by a Panasonic super-VHS PV-S7670 Pro Line Multiplex videocassette recorder with a 100 -ms digital videotimer (TEL Video Products model VC 436). The lateral view of the oral and pharyngeal structures was obtained. E-Z-HD barium sulfate powder for suspension was presented and patients were asked to swallow on instruction. The thin liquids, a mixture of water and barium, had a viscosity of $14 \mathrm{cP}$ and the nectar thick liquids, a mixture of thickened juice and barium, had a viscosity of $187 \mathrm{cP}$. The sequence of bolus presentation was as follows: first two swallows were $5 \mathrm{ml}$ thin liquid, next two swallows were $10 \mathrm{ml}$ thin liquid, next two swallows were $5 \mathrm{ml}$ nectar thick liquid, and the last two swallows were $10 \mathrm{ml}$ nectar thick liquid.

\section{Procedures for Recording Temporal Measurements}

Approval was obtained from Ohio University's internal review board for completing this investigation. Slowmotion frame-by-frame analysis was performed using the Adobe Premier Pro 1.5 and a $100 \mathrm{~ms}$ videotimer. A total of 533 swallows were analyzed based on the clarity of the pharyngeal structures. This investigation focused on two temporal measurements: STD and LCD. 
Stage transition duration refers to the time between bolus head passing the ramus of the mandible and the onset of maximum hyoid excursion [5]. LCD refers to the time between the first and last contact of the inferior surface of the epiglottis and the arytenoids [7]. Laryngeal closure in this investigation actually refers to laryngeal vestibule closure rather than true and/or false vocal folds closure. This terminology has been used in prior investigations [7, 8]. Reference points for obtaining these measurements included (1) bolus passing the ramus of the mandible, (2) initiation of maximum hyoid excursion, (3) initial contact between the arytenoids and the epiglottis, and (4) final contact between the arytenoids and epiglottis. The judges in this investigation were two graduate students who trained on temporal measures for at least 6 months for a previous investigation.

\section{Statistical Analysis}

A three-way analysis of variance (ANOVA) was used. The independent variables included the three groups, two bolus consistencies, and two bolus volumes. The dependent variables were the two temporal measures of pharyngeal swallowing, STD and LCD. Significance level was set at $p<0.05$.

\section{Results}

Intrajudge reliability was established by one of the authors (EO), who reanalyzed $23 \%$ of the total swallows. The interjudge reliability was established by a second judge (YK) who measured the temporal measurements for the same number of swallows. The Pearson correlation coefficient (r) was calculated for both sets of measurements. There was a significant relationship between the first and second judge's measurements for both STD $(r=0.97, p<0.05)$ and $\operatorname{LCD}(r=0.91, p$ B 0.05). There was also a significant relationship between the first and second measurements of the author for both STD $(r=0.97, p<0.05)$ and LCD $(r=0.91, p<0.05)$.

\section{Stage Transition Duration}

Aspirators exhibited the longest STD values across bolus consistency and volume compared to nonaspirators and control subjects. Poststroke patients (aspirators and nonaspirators) differed significantly from the control group with respect to mean $\operatorname{STD}[F(2,265)=26.65, p<0.01]$; however, despite a trend toward increased STD values in aspirators, the difference between aspirators and nonaspirators was not statistically significant.
Bolus consistency (thin versus nectar thick liquids) did not significantly affect $\operatorname{STD}[F(1,265)=0.27, p=0.87]$. Figure 1 shows the mean STD and the 95\% confidence interval across the three groups for the two bolus consistencies. STD increased across the three groups, with the control subjects exhibiting the shortest STD and the aspirators exhibiting the longest STD for both thin and nectar thick liquids.

Bolus volume did not significantly affect STD $[F(1$, $265)=1.08, p=0.30]$. Figure 2 shows the mean STD and the $95 \%$ confidence interval among the three groups for the two volumes. No interaction was observed between group and consistency or between group and volume.

\section{Laryngeal Closure Duration}

No significant differences were observed for mean LCD among the three groups $[F(2,265)=0.83, p=0.44]$. LCD did not differ for the two bolus consistencies $[F(1$, $265)=1.63, p=0.20]$ or the two bolus volumes $[F(1$, $265)=0.22, p=0.64]$. Figure 3 shows the mean LCD and the $95 \%$ confidence interval of the three groups for the two bolus consistencies.

Figure 4 shows the mean and the 95\% confidence interval for LCD among the three groups for the two bolus volumes. No interaction was observed between groups and consistencies or between groups and volumes for LCD.

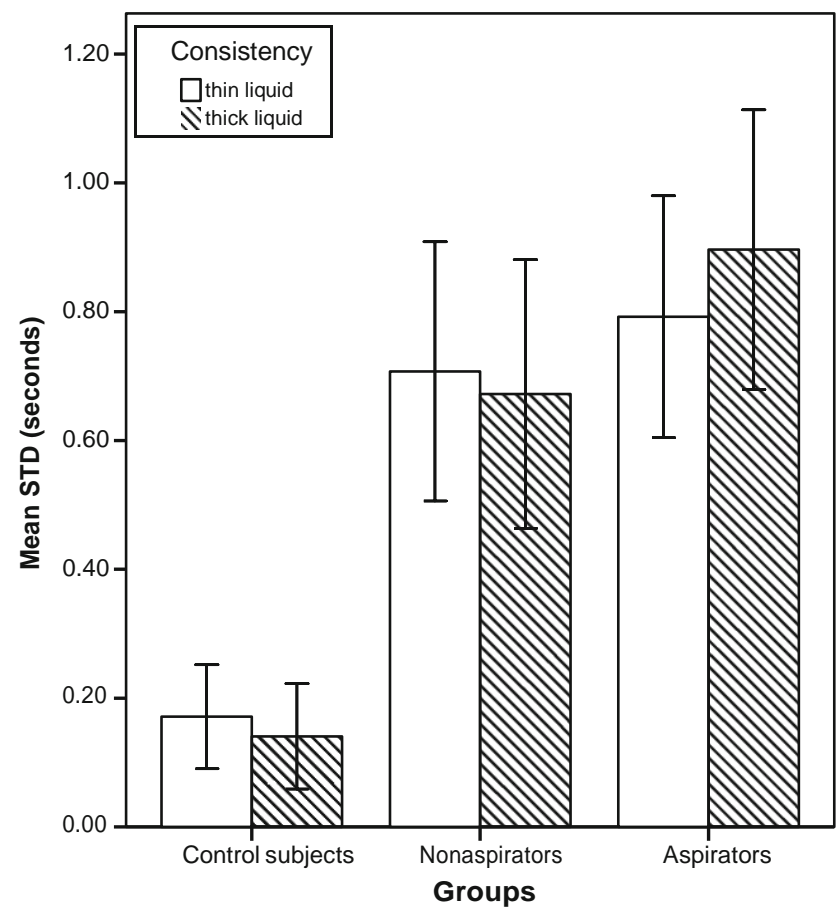

Fig. 1 Mean stage transition duration (STD) and 95\% confidence interval of the three groups for the two bolus consistencies 


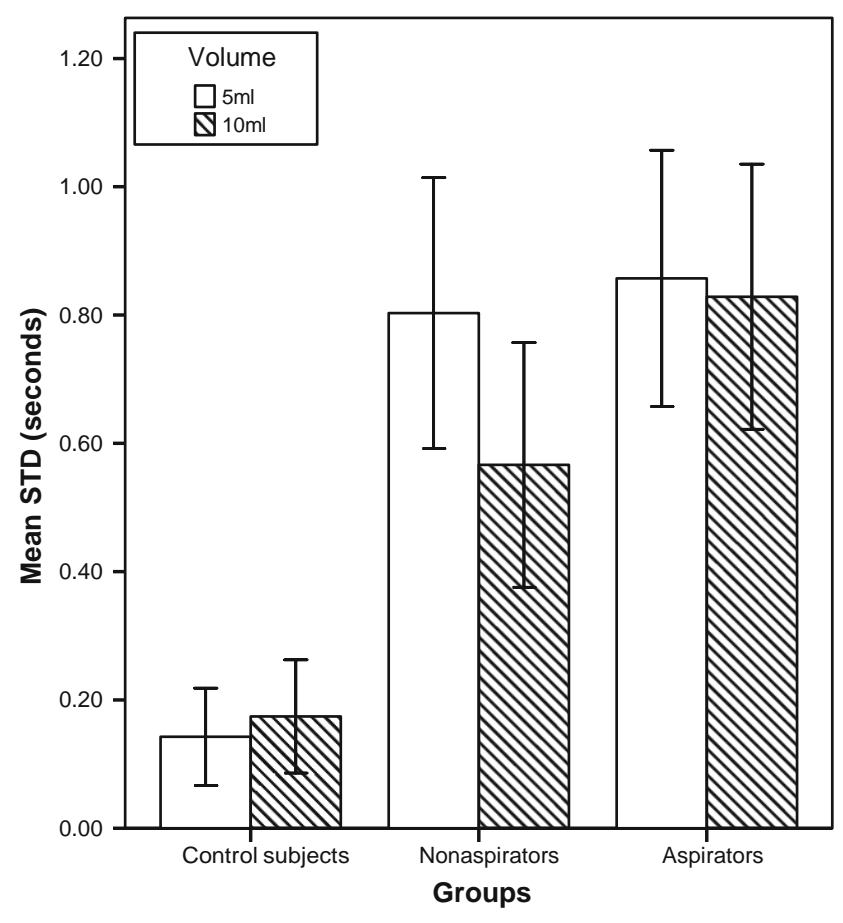

Fig. 2 Mean stage transition duration (STD) and 95\% confidence interval of the three groups for the two bolus volumes

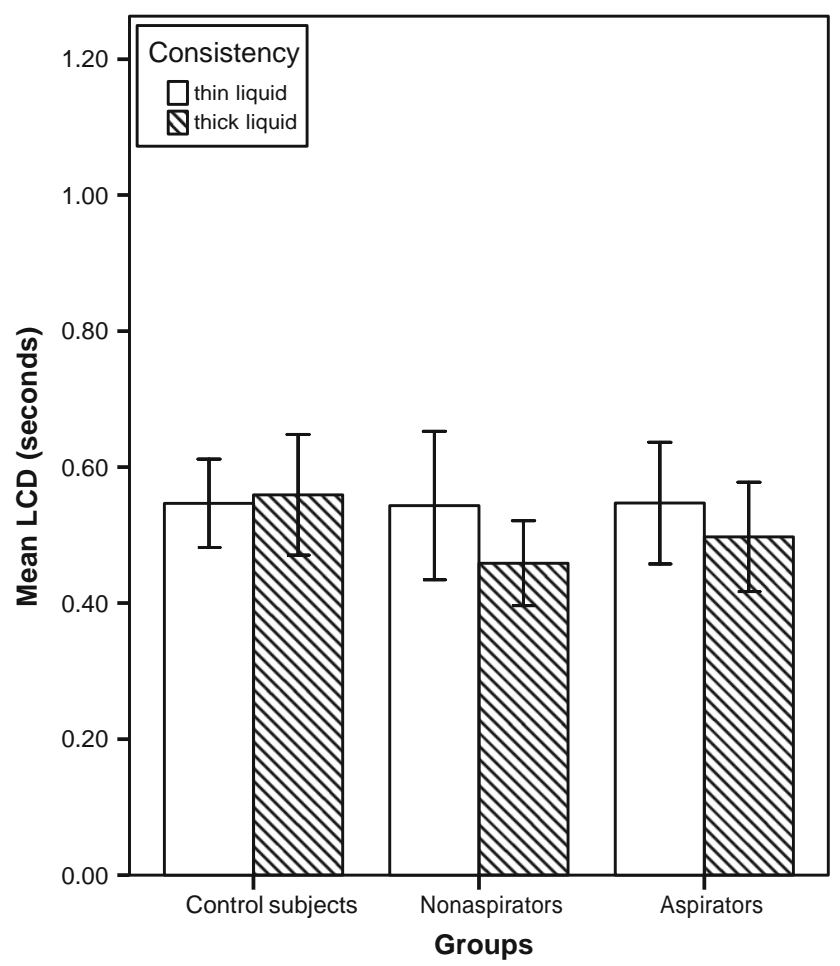

Fig. 3 Mean laryngeal closure duration and $95 \%$ confidence interval of the three groups for the two bolus consistencies

\section{Discussion}

Dysphagia is often seen in patients following an acute stroke [15] and signs and symptoms can manifest during the course

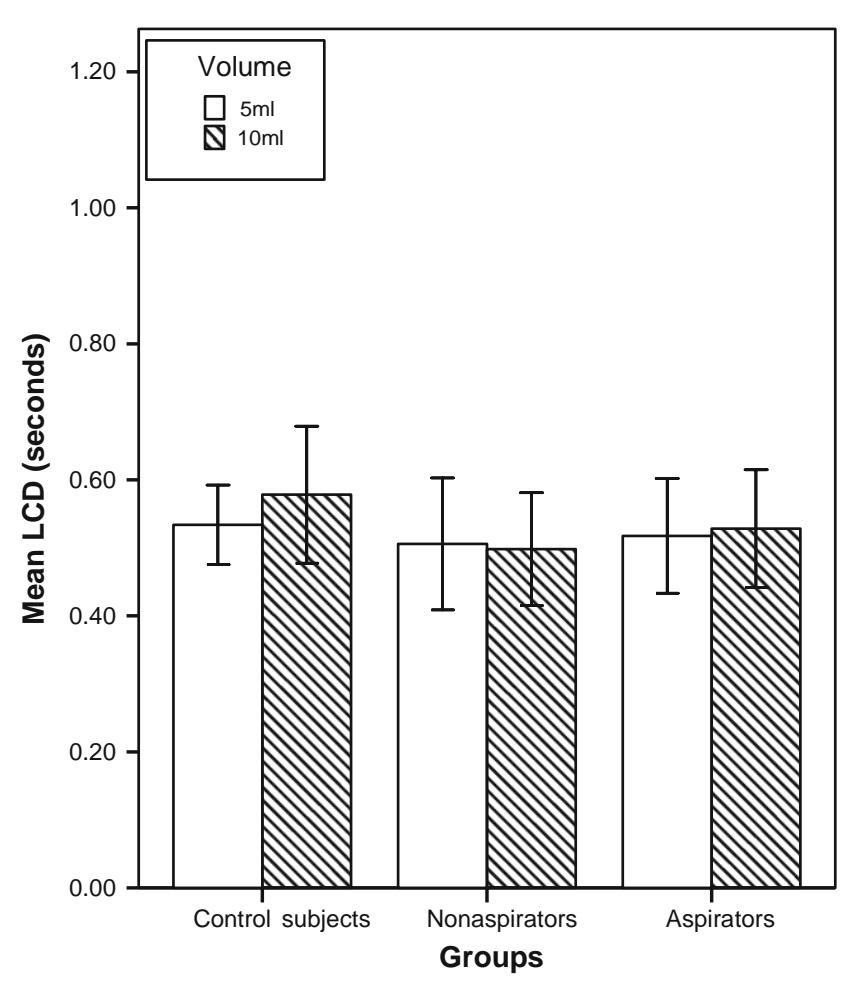

Fig. 4 Mean laryngeal closure duration and 95\% confidence interval of the three groups for the two bolus volumes

of illness [16]. Use of objective and quantitative temporal measurements may be helpful in the early identification of physiological impairments, such as delayed initiation of pharyngeal swallowing and reduced laryngeal closure, that could result in reduced efficiency and safety of swallowing.

The purpose of this investigation was to determine the effects of small changes in bolus consistency (thin and nectar thick liquids) and bolus volume (5 and $10 \mathrm{ml}$ ) on STD and LCD among three groups. The temporal measurements used in this investigation reflect important physiological events in pharyngeal swallowing: the initiation of hyoid excursion and the closure of the laryngeal vestibule. STD represents the transition between the oral and pharyngeal stages of swallowing, while LCD reflects the ability to protect the airway against the entry of the bolus during the swallow.

The results of this investigation showed that STD is significantly prolonged in the poststroke groups compared to the normal controls. Prolonged STD is a measure of delayed initiation of pharyngeal swallowing [5]. Longer STD values place the patient at an increased risk of aspiration because swallowing safety is compromised. In this investigation, differences between aspirating and nonaspirating stroke patients with respect to STD were not statistically significant, although the trend was clear: aspirators tend to have longer STDs than nonaspirators. Aspiration occurs for different reasons, one of which is a delayed 
swallow. Thus, different mechanisms responsible for aspiration could affect results. Our small sample is another potential reason for the trend not reaching significance. Kim and McCullough [6] concluded that STD is a good indicator of the potential risk of aspiration. They proposed that STD values could be used to monitor the progress of patients with dysphagia during intervention. In addition, the STD value may help the clinician determine whether treatment is to be initiated or whether follow-up visits are required. Clinically, STD will provide integral information regarding the physiological changes during intervention.

LCD reflects the integrity of the laryngeal protection mechanism in preventing the entry of the bolus into the laryngeal vestibule. In this investigation, LCD did not differ significantly among the three groups. Similar results between stroke patients who exhibited aspiration and those who did not [7] have been reported. Based on previous studies, LCD in isolation may not be a sensitive measure for indicating aspiration [17]. However, LCD can be interpreted with other measures such as STD to perhaps provide more robust and potentially useful results. A future study comparing aspirating swallows to nonaspirating swallows with a wider array of temporal measurements may provide a more robust method for predicting aspiration.

It has been established that changes in bolus consistency influence oral and pharyngeal swallowing [12]. This influence has been reported to occur when liquids are compared to semisolids [5, 12] and when nectar-thick liquids are compared to honey-thick liquids [18]. Our results, however, indicate that this is not true when comparing thin and nectar-thick liquids. Neither LCD nor STD was significantly altered. Perhaps the difference between nectar and honey is more robust in terms of sensory stimulation and diminishes the speed at which the bolus will travel more substantively. As more varieties of thick liquids are marketed with smaller incremental changes, these results raise important questions. Do we need more than two thick liquid viscosities? Will physiologic changes be robust enough when increases in viscosities are minimal? Research should address these questions in light of barium and therapeutic liquid products.

Changes in the bolus volume are also known to influence the temporal characteristics of pharyngeal swallowing $[11,12,19,20]$. This investigation examined the effects of two bolus volumes, 5 and $10 \mathrm{ml}$, on pharyngeal swallowing and found no differences in STD or LCD across the three groups. Again, the differences may not have been robust enough. Small increments in bolus volume did not affect LCD in healthy normal subjects and poststroke patients in prior research [7, 17]. As is the case with small changes in viscosity, we must continue to ask how small of a change in volume is necessary to change physiology. VFSEs are limited in time due to radiation exposure. Should patients really be tested with $1,3,5,10$, and $20 \mathrm{ml}$ boluses if research suggests that small changes in volume have no physiologic effect? Again, more research must be conducted to examine these differences not only for LCD and STD but for more temporal measures.

There were limitations to this investigation. We used radiographic swallowing examinations obtained from previous investigations. Thus, the sequence of bolus presentation was predetermined with respect to consistency and volume. Randomization of bolus could not be done as the sequence of bolus presentation in the VFSE clips was fixed and the authors were required to have knowledge of the same in order to correctly isolate the swallows for temporal analysis. In addition, the poststroke group was heterogeneous with respect to site of lesion and, thus, the effects of stroke on the physiology of swallowing were more varied in these patients. Other limitations of this investigation were the small number of subjects in all three groups and the limited age and gender matching.

In conclusion, temporal measurements of swallowing have provided insight into understanding how physiologic events are influenced by different bolus characteristics in poststroke patients. Our results indicate that smaller changes in bolus consistency and volume have little to no effect on the temporal measures of STD and LCD. These findings will add to the objective database that has been developed to understand the physiological impairments seen in dysphagia and should be carefully considered in regard to current clinical practice. Much more research is warranted in this important area.

Acknowledgment This research was supported in part by the Department of Veterans Affairs Rehabilitation, Research \& Development grants (C96-1143RA and C98-1442PA).

\section{References}

1. Logemann JA. Evaluation and treatment of swallowing disorders, 2nd ed. Austin, TX: Pro-Ed; 1998, p. 1-8.

2. Martino R, Foley N, Bhogal S, Diamant N, Speechley M, Teasell R. Dysphagia after stroke: incidence, diagnosis, and pulmonary complications. Stroke. 2005;36:2756-63.

3. Kim Y, McCullough GH, Asp CW. Temporal measurements of pharyngeal swallowing in normal populations. Dysphagia. 2005;20:290-6.

4. Kendall KA, McKenzie S, Leonard RJ, Goncalves MI, Walker A. Timing of events in normal swallowing: a videofluoroscopic study. Dysphagia. 2000;15:74-83.

5. Robbins J, Hamilton JW, Log GL, Kempster GB. Oropharyngeal swallowing in normal adults of different ages. Gastroenterology. 1992;103:823-9.

6. Kim Y, McCullough GH. Stage transition duration in patients poststroke. Dysphagia. 2007;22(4):299-305.

7. Power ML, Hamdy S, Singh S, Tyrrell PJ, Turnbull I, Thompson DG. Deglutitive laryngeal closure in stroke patients. J Neurol Neurosurg Psychiatry. 2007;78:141-6. 
8. Park T, Kim Y, Ko D, McCullough G. Initiation and duration of laryngeal closure during the pharyngeal swallow in post-stroke patients. Dysphagia. 2009;25:177-82.

9. Bisch EM, Logemann JA, Rademaker AW, Kahrilas PJ, Lazarus CL. Pharyngeal effects of bolus volume, viscosity, and temperature in patients with dysphagia resulting from neurologic impairment and in normal subjects. J Speech Hear Res. 1994;37: 1041-9.

10. Lazarus CL, Logemann JA, Rademaker AW, Kahrilas PJ, Pajak $\mathrm{T}$, Lazar L, et al. Effects of bolus volume, viscosity, and repeated swallows in non-stroke subjects and stroke patients. Arch Phys Med Rehabil. 1993;74(10):1066-70.

11. Logemann JA, Pauloski BR, Rademaker AW, Kahrilas PJ. Oropharyngeal swallow in younger and older women: videofluoroscopic analysis. J Speech Lang Hear Res. 2002;35:434-45.

12. Dantas RO, Kern MK, Massey BT, Dodds WJ, Kahrilas PJ, Brasseur JG, et al. Effect of swallowed bolus variables on oral and pharyngeal phases of swallowing. Am J Physiol. 1990;258:675-81.

13. McCullough GH, Wertz RT, Rosenbek JC. Sensitivity and specificity of clinical/bedside examination signs for detecting aspiration in adults secondary to stroke. J Commun Disord. 2001;34(1-2):55-72.

14. McCullough GH, Wertz RT, Rosenbek JC. Age, gender, size, consistency effects on swallowing function in adults between 21 and 99 years of age. Presented at the 10th Annual Meeting of Dysphagia Research Society, Alburquerque, NM, 2001.

15. Gordon C, Hewer RL, Wade DT. Dysphagia in acute stroke. $\mathrm{Br}$ Med J. 1987;295:411-4.
16. Smithard DG, O'Neill PA, England RE, Park CL, Wyatt R, Martin DF, et al. The natural history of dysphagia following a stroke. Dysphagia. 1997;12:188-93.

17. Kim Y, Park T. Age and gender differences of laryngeal closure duration during normal swallowing. Korean J Commun Disord. 2007; 12:521-31.

18. Logemann JA, Gensler G, Robbins J, Lindblad AS, Brandt D, Hind JA, et al. A randomized investigation of three interventions for aspiration of thin liquids in patients with dementia or Parkinson's disease. J Speech Lang Hear Res. 2008;51:173-83.

19. Logemann JA, Pauloski BR, Rademaker A, Cook B, Graner D, Milianti F, et al. Impact of the diagnostic procedure on outcome measures of swallowing rehabilitation in head and neck cancer patients. Dysphagia. 1992;7:179-86.

20. Logemann JA, Pauloski BR, Rademaker AW, Colangelo LA, Kahrilas PJ, Smith CH. Temporal and biomechanical characteristics of oropharyngeal swallow in younger and older men. J Speech Lang Hear Res. 2000;43:1264-74. 